\title{
Therapeutic Response to Dihydroartemisinin-Piperaquine for $P$. falciparum and $P$. vivax Nine Years after Its Introduction in Southern Papua, Indonesia
}

\author{
Jeanne Rini Poespoprodjo, ${ }^{1,2,3 \star}$ Enny Kenangalem, ${ }^{1,2}$ Johny Wafom, ${ }^{2}$ Freis Chandrawati, ${ }^{2}$ Agatha M. Puspitasari, ${ }^{4}$ Benedikt Ley, ${ }^{5}$ \\ Leily Trianty, ${ }^{4}$ Zoé Korten, ${ }^{5}$ Asik Surya, ${ }^{6}$ Din Syafruddin, ${ }^{4}$ Nicholas M. Anstey, ${ }^{5}$ Jutta Marfurt, ${ }^{5}$ Rintis Noviyanti, ${ }^{4}$ and Ric N. Price ${ }^{5,7}$ \\ ${ }^{1}$ Mimika District Hospital, Timika, Indonesia; ${ }^{2}$ Timika Malaria Research Programme, Papuan Health and Community Development Foundation, \\ Timika, Indonesia; ${ }^{3}$ Paediatric Research Office, Department of Child Health, Faculty of Medicine, Universitas Gadjah Mada/Dr. Sardjito Hospital, \\ Yogyakarta, Indonesia; ${ }^{4}$ Eijkman Institute for Molecular Biology, Jakarta, Indonesia; ${ }^{5}$ Global and Tropical Health Division, Menzies School of Health \\ Research and Charles Darwin University, Darwin, Australia; 'Indonesian Ministry of Health, National Malaria Control Program, Jakarta, Indonesia; \\ ${ }^{7}$ Centre for Tropical Medicine and Global Health, Nuffield Department of Clinical Medicine, University of Oxford, Oxford, United Kingdom
}

\begin{abstract}
Dihydroartemisinin-piperaquine (DHP) has been the first-line treatment of uncomplicated malaria due to both Plasmodium falciparum and Plasmodium vivax infections in Papua, Indonesia, since March 2006. The efficacy of DHP was reassessed to determine whether there had been any decline following almost a decade of its extensive use. An open-label drug efficacy study of DHP for uncomplicated $P$. falciparum and $P$. vivax malaria was carried out between March 2015 and April 2016 in Timika, Papua, Indonesia. Patients with uncomplicated malaria were administered supervised DHP tablets once daily for 3 days. Clinical and laboratory data were collected daily until parasite clearance and then weekly for 6 weeks. Molecular analysis was undertaken for all patients with recurrent parasitemia. A total of 129 study patients were enrolled in the study. At day 42 , the polymerase chain reaction-adjusted efficacy was $97.7 \%$ (95\% confidence intervals [Cl]: 87.4-99.9) in the 61 patients with $P$. falciparum malaria, and $98.2 \%$ [95\% Cl: 90.3-100] in the 56 patients with $P$. vivax malaria. By day $2,98 \%$ (56/57) of patients with $P$. falciparum and $96.9 \%(63 / 65)$ of those with $P$. vivax had cleared their peripheral parasitemia; none of the patients were still parasitaemic on day 3 . Molecular analysis of $P$. falciparum parasites showed that none $(0 / 61)$ had $\mathrm{K} 13$ mutations associated previously with artemisinin resistance or increased copy number of plasmepsin 2-3 (0/61). In the absence of artemisinin resistance, DHP has retained high efficacy for the treatment of uncomplicated malaria despite extensive drug pressure over a 9-year period.
\end{abstract}

\section{INTRODUCTION}

Over the last 50 years, Plasmodium falciparum has developed resistance to chloroquine (CQ), mefloquine, sulphadoxinepyrimethamine, piperaquine, and artemisinin, with the earliest evidence of resistance for all of these drugs documented in the Greater Mekong Subregion (GMS). By contrast, the epicenter of antimalarial drug resistance in Plasmodium vivax is on the island of Papua. In southern Papua, Indonesia, both $P$. falciparum and $P$. vivax malaria are equally prevalent. ${ }^{1,2}$ Clinical trials conducted in 2004 highlighted a very high risk of treatment failure following treatment with locally recommend antimalarial regimens. Within 28 days, $48 \%$ of patients with $P$. falciparum had failed treatment with $\mathrm{CQ}$ plus sulfadoxinepyrimethamine $(\mathrm{CQ}+\mathrm{SP})$ and $65 \%$ of those with $P$. vivax had failed treatment with $C Q$ monotherapy. ${ }^{3}$ In view of these findings, the first-line treatment of uncomplicated malaria due to either $P$. falciparum or $P$. vivax was changed to dihydroartemisinin-piperaquine (DHP) in March 2006. At the time of introduction of DHP, the risk of recurrent parasitemia within 42 days was $4.1 \%$ following $P$. falciparum and $10 \%$ following $P$. vivax malaria. ${ }^{4}$

Over the last 10 years, there has been growing concern regarding the emergence of artemisinin-resistant parasites in the GMS. ${ }^{5-7}$ The spread of these parasites to other locations has potential to undermine the global policy of artemisininbased combination therapy (ACT) for uncomplicated malaria. More recently, there have been reports of declining efficacy of the partner drug piperaquine in areas where the prevalence of artemisinin resistance is high. ${ }^{8}$ The spread of these

*Address correspondence to Jeanne Rini Poespoprodjo, Timika Malaria Research Programme, Papuan Health and Community Development Foundation, Timika, Indonesia. E-mail: didot2266@ yahoo.com multidrug-resistant parasites eastward across the Indonesian archipelago poses a significant threat to the national antimalarial policy. In Papua, where DHP has been used extensively over the last 9 years, a clinical study was undertaken to assess whether it still retained excellent efficacy.

\section{MATERIALS AND METHODS}

Study site. The study was carried out in Timika, southern Papua, Indonesia, between March 2015 and April 2016. The epidemiology of this area has been described previously. 1,9,10 In brief, malaria is restricted to lowland areas where it is associated with three mosquito vectors: Anopheles koliensis, $A n$. farauti, and An. punctulatus. The area has unstable malaria transmission. A household survey conducted in the same area in 2013 showed that malaria prevalence was $13.1 \%$, with $P$. falciparum and $P$. vivax accounting for $5.9 \%$ and $6.0 \%$ of malaria, respectively. ${ }^{11}$

DHP is available, free of charge, from government health facilities and selected private-sector health facilities. Local protocol dictates that DHP is prescribed to patients with malaria confirmed by either rapid diagnostic test or microscopy. Local surveillance data showed that over 10 years, more than 485,000 doses of DHP have been administered to a population of 220,000 people. A cross-sectional survey undertaken in 2013 estimated that approximately $67 \%$ of individuals attend public health clinics, where DHP is available, for treatment of febrile illness. ${ }^{11,12} \mathrm{CQ}$ and SP were available in a few private pharmacies and their use has declined to less than $5 \%$ following policy change in March 2006.

Study design. This was a prospective open-label drug efficacy study of dihydroartemisinin-piperaquine for $P$. falciparum and $P$. vivax malaria in children and adults with uncomplicated symptomatic malaria. The study was based 
on the 2009 World Health Organization antimalarial drug therapeutic efficacy tests ${ }^{13}$ with 42 days of follow-up.

Patients. Patients were eligible to enroll in the study if they were aged between 1 and 65 years, weighed more than $5 \mathrm{kgs}$, had a fever or history of fever in the preceding 24 hours, with slide-confirmed malaria with parasitemia of more than $1,000 / \mu \mathrm{L}$ asexual parasites for $P$. falciparum and more than $250 / \mu \mathrm{L}$ asexual parasites for $P$. vivax. Pregnant and lactating women, patients with signs of severe malaria, ${ }^{14}$ or those presenting with severe malnutrition or significant comorbidities were all excluded. Informed consent was obtained from patients aged more than 18 years old and in those less than 13 years old, from their parent or guardian. Children aged between 13 and $<18$ years were asked for their assent in the presence of their parent or guardian. If patients were illiterate, consent was obtained in the presence of a literate witness. All patients were provided with an information sheet in Indonesian language.

Study procedures. On admission, a standardized questionnaire was completed to record demographic information and details of the patients' symptoms, prior antimalarial medication, and their clinical examination. Axillary temperature was measured using a digital electronic thermometer. Venous or capillary blood was taken at enrolment for malaria smear, hemoglobin concentration, and parasite genotyping. Hemoglobin was measured using a portable photometer (HemoCue $^{\mathrm{TM}} \mathrm{Hb} 201+$; Ångelholm, Sweden). Glucose 6 phosphate dehydrogenase (G6PDd) status was measured at enrolment using the Fluorescent Spot Test method. ${ }^{15}$

Patients were examined daily for the first 3 days of DHP treatment and then weekly for 6 weeks. Patients were also encouraged to return to the clinic on any other day if they felt unwell. At each visit, a physical examination was performed, the symptom questionnaire completed, and blood taken for blood film examination and measurement of hemoglobin concentration.

Parasitemia was determined by microscopic examination of Giemsa-stained thick blood films, with parasites counted per 200 white blood cells (WBC) and peripheral parasitemia calculated assuming a white cell count of $7,300 / \mu \mathrm{L}$. Slides were considered negative after review of 400 high-power fields. A thin smear was also examined to confirm parasite species and used for quantification if the parasitemia was greater than 200 per 200 WBC. All slides were read by a certified microscopist and cross-checked by a second experienced microscopist. In cases where readings were discordant, the slides were reread by a third microscopist and a consensus reached.

DNA from venous or capillary blood samples collected in ethylene diamine tetraacetic acid-coated Vacutainers or Microtainers $^{\mathrm{TM}}$ at enrolment and again on the day of failure was extracted using the QIAamp DNA mini kit (Qiagen, Chadstone Centre, Victoria, Australia) as per the manufacturer's instructions. Speciation was done by using an 18sRNA gene-based polymerase chain reaction (PCR) method for the detection of four different malaria species. ${ }^{16,17}$ For patients with recurrent $P$. falciparum infection, pre- and posttreatment isolates were genotyped using $m s p 1, m s p 2$, and glurp as described previously. ${ }^{18,19}$ The presence of polymorphisms in the propeller domains of the $P$. falciparum kelch (K13) gene, associated with artemisinin derivatives resistance, was determined by Sanger sequencing using the WWARN protocol. ${ }^{20}$ Plasmepsin 2-3 copy number variants, associated with piperaquine resistance, were also examined in all samples as described previously. ${ }^{21-23}$
Treatment. Dihydroartemisinin-piperaquine (containing $40 \mathrm{mg}$ dihydroartemisinin and $320 \mathrm{mg}$ piperaquine) was administered once daily for 3 days as a dose-per-weight regimen with a target dose of 2.25 and $18 \mathrm{mg} / \mathrm{kg}$ of dihydroartemisinin and piperaquine, respectively, according to the National Guidelines. ${ }^{24}$ In addition, patients with $P$. vivax malaria were prescribed primaquine $(0.5 \mathrm{mg} / \mathrm{kg} /$ day for 14 days) commencing on day 28 of the study follow-up. All doses of DHP, but not primaquine, were administered under direct supervision and the patients observed for 30 minutes for adverse reactions or vomiting. Any patient vomiting their medication within this period was re-administered the same dose of DHP and observed for an additional 30 minutes. If the patient vomited again, then they were withdrawn from the study and hospitalized for artesunate intravenous therapy.

Patients with therapeutic failure within 28 days of initial treatment ${ }^{25}$ were treated according to local policy with a 7-day course of oral quinine $(10 \mathrm{mg} / \mathrm{kg}$ body weight per dose, three times daily) plus doxycycline ( $2 \mathrm{mg} / \mathrm{kg}$ body weight per day, divided in two dose) in patients older than 8 years or clindamycin ( $5 \mathrm{mg} / \mathrm{kg}$ body weight per dose, three times per day) in children younger than 8 years. Patients with treatment failure after 28 days were retreated with DHP.

Endpoints. The primary endpoints of the study were the PCR-adjusted risk of recurrence of $P$. falciparum and the unadjusted risk of $P$. vivax recurrence. Secondary endpoints included the proportion of patients still parasitaemic on days 1 , 2 , and 3 , posttreatment gametocyte carriage, and hematological recovery.

Statistical analysis. Data were double entered and validated using EpiData 3.02 software (EpiData Association, Odense, Denmark) and analysis performed using SPSS for Windows (SPSS Inc., Chicago, IL). The Mann-Whitney $U$ test or Kruskal-Wallis method were used for nonparametric comparisons and Student's $t$ test or one-way analysis of variance for parametric comparisons. For categorical variables, percentages and corresponding 95\% confidence intervals $(95 \% \mathrm{Cl})$ were calculated using Wilson's method. Proportions were examined using $x^{2}$ with Yates' correction or by Fisher's exact test. The cumulative risk of failure was assessed by survival analysis using the Kaplan-Meier method. Anyone lost to follow up or representing with a different outcome were censored on their last day of follow-up and regarded as not being treatment failures. For determining the risk of $P$. falciparum recrudescence, all early treatment failures and homologous recrudescences were considered failures.

Ethics. The study was approved by the Eijkman Institute Research Ethics Commission, Jakarta, Indonesia (Ref: 014/ElIRB/XII/2014), and the Human Research Ethics Committee of the NT Department of Health \& Families and Menzies School of Health Research, Darwin, Australia (HREC-2015-2342). Informed consent was obtained from all adult participants and from parents of children. The trial was registered with the clinical trials website (http://www.clinicaltrials.gov/ct) as NCT 02353494.

\section{RESULTS}

Between March 2015 and April 2016, 4,269 patients with fever were assessed by the health facility staff, of whom 1,039 (24\%) were positive for malaria (513 P. falciparum, 479 
P. vivax, and 27 mixed species malaria). Of these patients, 329 (32\%) were screened by the study team for enrolment criteria. A total of 281 patients were eligible for the study, of whom 129 (45.9\%) gave consent to participate in the study (61 $P$. falciparum and $68 P$. vivax malaria) (see Figure 1 and Table 1). Of 48 patients excluded from the study, 46 were just visiting and two had comorbidities. Most of the patients who declined to be enrolled in the study were uncomfortable with making decisions without prior consultation with other family members. Molecular analysis at enrolment was possible in all but one patient, with the species of infection determined by PCR in concordance with that from microscopy.

Five patients $(3.9 \%)$ vomited their medication after the first dose, but tolerated the repeat dose. The median total dose of dihydroartemisinin administered was $7 \mathrm{mg} / \mathrm{kg}$ (range 5.4-10.6) and that of piperaquine was $57 \mathrm{mg} / \mathrm{kg}$ (range 43.2-84.7). In total, one male patient with $P$. falciparum was G6PD deficient and another male with $P$. vivax had intermediate G6PD activity; primaquine was not prescribed to either of these patients.

Therapeutic response. In total, four patients had recurrent parasitemia during the follow-up period (three with $P$. falciparum and one with $P$. vivax). The overall unadjusted efficacy at day 42 was $98.2 \%$ [95\% Cl: 90.3-100].

Of the 61 patients with $P$. falciparum infection, 43 (70.5\%) completed 42 days of follow-up and 41 had an adequate clinical and parasitological response. Two patients had asymptomatic recurrent $P$. falciparum parasitemia recorded on day 42: a 15-year-old female treated with $48.8 \mathrm{mg} / \mathrm{kg}$ of piperaquine with reinfection and a 52-year-old male treated with $53.3 \mathrm{mg} / \mathrm{kg}$ of piperaquine with recrudescent infection. The unadjusted efficacy for $P$. falciparum at day 42 was $95.3 \%$ [95\% Cl: 84.2-99.4] and after adjusting by PCR, this rose to
97.7\% [95\% Cl: 87.4-99.9]. Of the 68 patients with $P$. vivax infection, 56 (82.3\%) completed follow-up. Two patients had recurrent parasitemia on day 35: a 2-year-old boy with $P$. vivax infection in whom the parasite genotype could not be determined because insufficient blood was taken at enrolment and an 18-year-old female with symptomatic $P$. falciparum infection. The unadjusted efficacy for $P$. vivax at day 42 was 98.2\% [95\% Cl: 90.4-100]; Table 2.

Parasite clearance was rapid following treatment in both $P$. falciparum and $P$. vivax malaria. Within 24 hours, $62.7 \%(37 / 59)$ of patients with $P$. falciparum and $74.6 \%$ (50/67) of those with $P$. vivax were aparasitemic, and by 48 hours, this had risen to $98.2 \%(56 / 57)$ and $96.9 \%$ (63/65), respectively. None of these patients were parasitemic on day 3 .

Gametocyte carriage following treatment. Gametocytemia was present at enrolment in 3.3\% (2/61) of patients infected with $P$. falciparum and $26.5 \%(18 / 68)$ with $P$. vivax malaria. The proportion of patients with patent gametocytemia in those with $P$. falciparum infection was $10.2 \%(6 / 59)$ at 24 hours, $12.3 \%(7 / 57)$ at 48 hours, and $5.9 \%(3 / 51)$ at day 7 . In patients with $P$. vivax malaria, the proportion with gametocytes fell to $6 \%(4 / 67)$ by 24 hours, $4.6 \%$ (3/65) at 48 hours, and $1.5 \%(1 / 65)$ at day 7 . One patient had $P$. falciparum gametocytes detected at day 14 , and one patient had $P$. vivax gametocytes detected at day 21 . None of the patients had gametocytes after day 21 .

Hemoglobin reduction after treatment. Mean hemoglobin concentration at enrolment was $13.8 \mathrm{~g} / \mathrm{dL}[95 \% \mathrm{Cl}$ : 13.1-14.5] in patients with $P$. falciparum and $13.2 \mathrm{~g} / \mathrm{dL}[95 \%$ Cl: 12.6-13.8] in those with $P$. vivax (Table 1). The mean reduction in hemoglobin concentration at day 14 was $1.65 \mathrm{~g} / \mathrm{dL}$ [95\% Cl: 1.14-2.15] in P. falciparum and $0.83 \mathrm{~g} / \mathrm{dL}[95 \% \mathrm{Cl}$ : $0.31-1.33]$ in $P$. vivax malaria $(P=0.661)$.

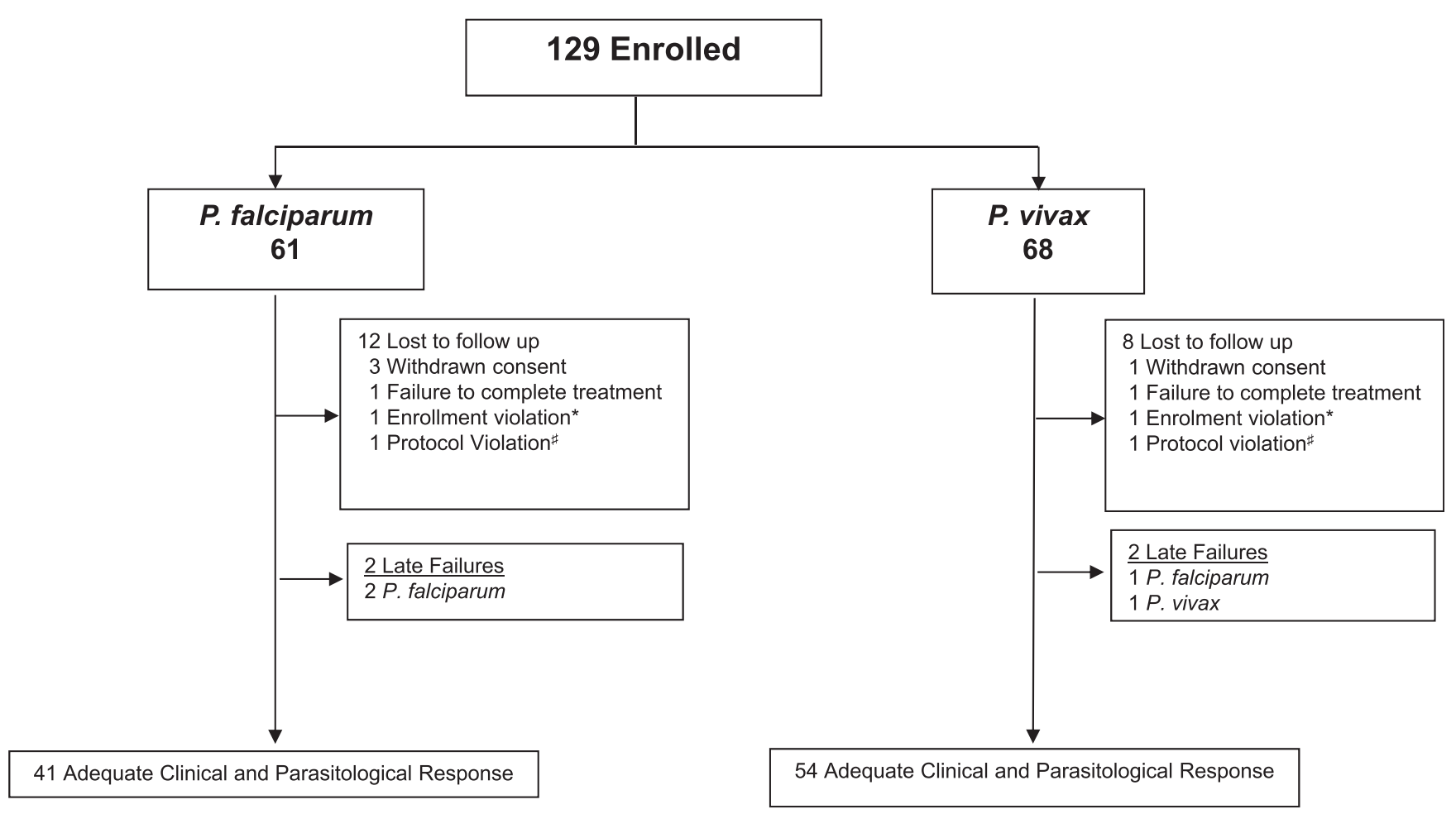

Figure 1. Trial Profile. 
TABLE 1

Baseline characteristics of uncomplicated malaria study patients

\begin{tabular}{|c|c|c|c|}
\hline & Plasmodium falciparum & Plasmodium vivax & $P$ value \\
\hline Number of subjects enrolled & 61 & 68 & \\
\hline Males \% $(N)$ & $62.3(38)$ & $50(34)$ & 0.214 \\
\hline \multicolumn{4}{|l|}{ Age (years) } \\
\hline Median [range], years & $23.5[4.3-63.9]$ & $18.3(1.8-50)$ & 0.075 \\
\hline 1 to $<5$ years $\%(n)$ & $1.6(1)$ & $1.5(1)$ & \\
\hline 5 to $<15$ years $\%(n)$ & $18.0(11)$ & $30.9(21)$ & 0.241 \\
\hline 15 to 65 years $\%(n)$ & $80.3(49)$ & $67.6(46)$ & \\
\hline \multicolumn{4}{|l|}{ Temperature $\left({ }^{\circ} \mathrm{C}\right)$} \\
\hline$>37.5 \%(n)$ & $52.5(32)$ & 48. (33) & 0.725 \\
\hline Mean $(95 \% \mathrm{Cl})$ & $37.8(37.5-38.1)$ & $37.8(37.5-38.2)$ & 0.033 \\
\hline \multicolumn{4}{|l|}{ Hemoglobin (g/dL) } \\
\hline Mean $(95 \% \mathrm{Cl})$ & $13.8(13.1-14.5)$ & $13.2(12.6-13.8)$ & 0.699 \\
\hline$<10 \mathrm{~g} / \mathrm{dL} \%(n / N)$ & $5.2(3 / 58)$ & $6.2(4 / 65)$ & 1.000 \\
\hline $\begin{array}{l}\text { Geometric mean }(95 \% \mathrm{Cl}) \text { parasite count } \\
\text { per } \mu \mathrm{L} \text { blood }\end{array}$ & $12,088(9,897-14,764)$ & $9,897(7,331-10,938)$ & 0.994 \\
\hline
\end{tabular}

$\mathrm{Cl}=$ confidence intervals.

Adverse events. No severe adverse events were reported associated with treatment. Twenty-four hours following treatment, $21.8 \%(27 / 124)$ of the patients had nausea (8), dyspepsia (2), dizziness (13), urticaria (1), and weakness (3). At 48 hours, 9.9\% (12/121) patients had mild nausea. All symptoms were resolved quickly. One case with urticaria was not associated with DHP, but due to mild food allergy.

K13 genes and plasmepsin 2-3 amplifications in $\boldsymbol{P}$. falciparum. Molecular analysis could be undertaken in all $P$. falciparum samples, with no mutations in the K13 gene $(0 / 65)$ or increased copy number of plasmepsin 2-3 (0/74).

\section{DISCUSSION}

In March 2006, Indonesia was one of the first countries to adopt a unified first-line policy of ACT for the treatment of uncomplicated malaria due to any species of malaria. It was also the first country to adopt DHP as its preferred choice of ACT, a decision made in view of its high efficacy and prolonged posttreatment prophylaxis associated with piperaquine, the slowly eliminated partner drug. ${ }^{4}$ Over the ensuing decade, DHP has been used extensively throughout the country. This recent therapeutic efficacy study in the eastern province of Papua reassuringly confirms that, despite the high prevalence of artemisinin and emerging piperaquine resistance in the GMS, DHP retains high efficacy against uncomplicated $P$. falciparum and $P$. vivax malaria after 9 years of use in this region.

The PCR-adjusted efficacy in patients with $P$. falciparum malaria was greater than $97 \%$, similar to that reported in $2005 .{ }^{4}$ The efficacy of DHP against $P$. vivax was almost
$100 \%$, with only one of 56 patients $(1.8 \%)$ having a recurrent infection on day 35 , compared with an efficacy of $90 \%$ reported in $2005 .{ }^{4}$ Compared with our original efficacy study in 2005, the baseline parasitemias in the present study were 3-4 fold higher and the gametocyte carriage 2-7 fold lower. The high gametocyte carriage at enrolment in 2005 likely reflects the poor efficacy of the first-line treatment before policy change in 2006 , with almost $20 \%$ of the patients reporting a history of malaria in the preceding month. ${ }^{3}$ With the wide deployment of highly efficacious treatment, the proportion of patients partially treated and reporting recent malaria has fallen substantially. ${ }^{11,12}$

Patients were only assessed once per day precluding precise estimation of the slope of the parasite clearance curves. ${ }^{26}$ However, the proportion of parasitemic patients fell rapidly, with more than $98 \%$ aparasitemic by 48 hours and none of the patients remaining parasitemic at 72 hours. Molecular analysis confirmed that none of the parasites had K13 gene mutations that have been associated with artemsinin resistance or amplification of the plasmepsin 2-3 gene cluster that have been associated with piperaquine resistance.

Resistance to artemisinin derivatives for the treatment of $P$. falciparum malaria was first described in western Cambodia in $2008^{27}$ and has been reported subsequently in Thailand, Myanmar, Laos, and Vietnam. ${ }^{6,28,29}$ In Cambodia, where the prevalence of artemisinin resistance is greatest, recent clinical trials have revealed that the efficacy of DHP for $P$. falciparum has fallen to less than $80 \%$, with almost half of the patients still parasitemic at 72 hours. ${ }^{30}$ Reassuringly, in the same study DHP retained good efficacy against $P$. vivax. ${ }^{30}$

Piperaquine was first synthesized in the 1960s and used extensively in China and Indochina as prophylaxis and

TABLE 2

The cumulative risk of treatment failure according to initial species of infection

\begin{tabular}{lcc}
\hline & Plasmodium falciparum [95\% Cl] & Plasmodium vivax [95\% Cl] \\
\hline Early treatment failure \% & 0 & 0 \\
$\begin{array}{l}\text { Risk of treatment failure at day 28 } \\
\text { Risk of recurrence with the same species }\end{array} \quad$ o & 0 \\
$\quad$ at day 42 & $4.7[0.6-15.8]$ & $1.8[0-9.7]$ \\
$\begin{array}{l}\text { Risk of recurrence by day 42-PCR } \\
\quad \text { adjusted }\end{array}$ & $2.3[0.1-12.3]$ & $-{ }^{*}$ \\
\hline
\end{tabular}

$\mathrm{Cl}=$ confidence intervals; $\mathrm{PCR}=$ polymerase chain reaction

* One recurrent infection with $P$. vivax, which had insufficient blood to genotype. 
treatment over the next 20 years. Within a decade, high rates of treatment failure were observed and piperaquine monotherapy was discontinued. The drug was removed from the pharmacopeia until it was reintroduced in 1990s in combination with dihydroartemisinin, trimethoprim, and primaquine phosphate. ${ }^{31}$ The recommended combination has subsequently rationalized to DHA plus piperaquine which has been available outside of China since the start of the millennium. The significant mismatch in the pharmacokinetic profiles of these two drug components has raised concerns of exposing parasites to subtherapeutic concentrations of piperaquine monotherapy in the 2-6 weeks following treatment, particularly where the risk of reinfection is high. It is feared that this mismatch will foster the emergence and spread of drug-resistant parasites. Indeed in this respect, it is interesting to note that in an area where artemisinin resistance has emerged the efficacy of the combination fell quickly. ${ }^{8,21,30}$

The results from this Papuan study are reassuring and suggest that in the absence of artemisinin resistance, the ACT regimen protects against de novo emergence of resistance. In Cambodia, amplification of the plasmepsin 2-3 gene cluster has been identified as an important molecular determinant of piperaquine resistance in $P$. falciparum. ${ }^{22}$ Our molecular analysis reveals that neither of these polymorphisms is present in Timika.

It is likely that the development of artemisinin resistance in Cambodia has been driven by high rates of self-treatment via private sector and unregulated use of antimalarial agents, including poor ACT quality. In Indonesia, DHP procurement is highly regulated by the Indonesian Ministry of Health, and the drug is only available at government health facilities and selected private-sector facilities, which are able to confirm the diagnosis of malaria by microscopy or rapid diagnostic test. Hence, DHP is generally only prescribed to those with confirmed malaria and this degree of control may have contributed to the sustained efficacy of the drug in this region.

In conclusion, despite intense use of DHP over almost a decade in a region of mesoendemic malaria, there is no evidence for the presence of either artemisinin or piperaquine resistance and the combination regimen retains excellent efficacy. However, in view of the highly mobile population and potential spread of artemisinin resistance from the GMS, vigilance for declining efficacy is warranted.

Received August 20, 2017. Accepted for publication October 20, 2017.

Published online January 15, 2018.

Acknowledgments: We thank the study participants for their significant contribution to the study and the excellent work of the field and laboratory staff of the Papuan Health and Community Development Foundation. We are grateful to Mimika District Health Authority, Lembaga Pengembangan Masyarakat Amungme Kamoro, for facilitating the study and thank Mr. Erens Meokbun (The Head of District Health Authority) and the staff of Puskesmas Timika. We also thank Dr. Dorina Bustos (SEARO), Dr. Anand Joshi (WHO Indonesia), Dr. Leonard Ortega (SEARO), and Dr. Elvieda Sariwati (Malaria Directorate, Indonesian Ministry of Health) for supporting the study and giving helpful and insightful suggestions. We also thank Professor Rick Fairhurst and Dr. Megan Ansbro for their advice on the plasmepsin molecular assay.

Financial support: The study was funded by the WHO SEARO and the Wellcome Trust. J. R. P. is a Wellcome Trust Research Training Fellow (099875/Z/12/Z) and R. N. P. is a Senior Wellcome Trust Senior Fellow in Clinical Science (200909). The DFAT Tropical Disease Research Regional Collaboration Initiative in Papua, Indonesia, is supported by the Australian Government (72904).

Authors' addresses: Jeanne Rini Poespoprodjo, Mimika District Hospital, Timika, Papua, Indonesia, Timika Malaria Research Programme, Papuan Health and Community Development Foundation, Papua, Indonesia, and Paediatric Research Office, Department of Child Health, Faculty of Medicine, Universitas Gadjah Mada/Dr. Sardjito Hospital, Yogyakarta, Indonesia, E-mail: didot2266@yahoo. com. Enny Kenangalem, Mimika District Hospital, Timika, Papua, Indonesia, and Timika Malaria Research Programme, Papuan Health and Community Development Foundation, Papua, Indonesia, E-mail: ennykenangalem@yahoo.com. Johny Wafom and Freis Chandrawati, Timika Malaria Research Programme, Papuan Health and Community Development Foundation, Papua, Indonesia, E-mails: wafomj@ yahoo.co.id and freiscandrawati@yahoo.com. Agatha M. Puspitasari, Leily Trianty, Din Syafruddin, and Rintis Noviyanti, Eijkman Institute for Molecular Biology, Jakarta, Indonesia, E-mails: agatha@eijkman. edu.au, leily@eijkman.go.id, din@eijkamn.go.id, and rintis@eijkamn. go.id. Benedikt Ley, Zoé Korten, Nicholas M. Anstey, and Jutta Marfurt, Global and Tropical Health Division, Menzies School of Health Research and Charles Darwin University, Darwin, Australia, E-mails: benedikt.ley@menzies.edu.au, zoe.korten@menzies.edu.au, nicholas. anstey@menzies.edu.au, and jutta.marfurt@menzies.edu.au. Asik Surya, National Malaria Control Program, Indonesian Ministry of Health, Jakarta, Indonesia, E-mail: asiksurya@yahoo.com. Ric N. Price, Global and Tropical Health Division, Menzies School of Health Research and Charles Darwin University, Darwin, Australia, and Centre for Tropical Medicine and Global Health, Nuffield Department of Clinical Medicine, University of Oxford, Oxford, United Kingdom, E-mail: rprice@menzies.edu.au.

This is an open-access article distributed under the terms of the Creative Commons Attribution License, which permits unrestricted use, distribution, and reproduction in any medium, provided the original author and source are credited.

\section{REFERENCES}

1. Karyana M et al., 2008. Malaria morbidity in Papua, Indonesia, an area with multidrug resistant Plasmodium vivax and Plasmodium falciparum. Malar J 7: 148.

2. Elyazar IR, Hay SI, Baird JK, 2011. Malaria distribution, prevalence, drug resistance and control in Indonesia. Adv Parasitol 74: $41-175$.

3. Ratcliff $A$ et al., 2007. Therapeutic response of multidrug-resistant Plasmodium falciparum and $P$. vivax to chloroquine and sulfadoxine-pyrimethamine in southern Papua, Indonesia. Trans $R$ Soc Trop Med Hyg 101: 351-359.

4. Ratcliff $A$, Siswantoro $H$, Kenangalem E, Maristela R, Wuwung RM, Laihad F, Ebsworth EP, Anstey NM, Tjitra E, Price RN, 2007. Two fixed-dose artemisinin combinations for drug-resistant falciparum and vivax malaria in Papua, Indonesia: an open-label randomised comparison. Lancet 369: 757-765.

5. Dondorp AM et al., 2009. Artemisinin resistance in Plasmodium falciparum malaria. N Engl J Med 361: 455-467.

6. Ashley EA et al., 2014. Spread of artemisinin resistance in Plasmodium falciparum malaria. N Engl J Med 371: 411-423.

7. WHO, 2016. Artemisinin and Artemisinin Based Combination Therapy Resistance: Status Report. Geneva, Switzerland: World Health Organization.

8. Amaratunga $C$ et al., 2016. Dihydroartemisinin-piperaquine resistance in Plasmodium falciparum malaria in Cambodia: a multisite prospective cohort study. Lancet Infect Dis 16: 357-365.

9. Poespoprodjo JR, Fobia W, Kenangalem E, Lampah DA, Hasanuddin A, Warikar N, Sugiarto P, Tjitra E, Anstey NM, Price RN, 2009. Vivax malaria: a major cause of morbidity in early infancy. Clin Infect Dis 48: 1704-1712.

10. Kenangalem E, Karyana M, Burdarm L, Yeung S, Simpson JA, Tjitra E, Anstey NM, Poespoprodjo JR, Price RN, Douglas NM, 2016. Plasmodium vivax infection: a major determinant of severe anaemia in infancy. Malar J 15: 321. 
11. Thio F, Indrawanti R, Price R, 2013. The Timika Household Survey 2 Databases: Changes in Treatment Seeking Behaviour. Timika, Papua: Timika Research Unit.

12. Devine A, Kenangalem E, Burdam FH, Anstey NM, Poespoprodjo JR, Price R, Yeung S, 2018. Treatment-seeking behavior after the implementation of a unified policy of dihydroartemisininpiperaquine for the treatment of uncomplicated malaria in Papua, Indonesia. Am J Trop Med Hyg 98: 543-550.

13. WHO, 2009. Methods for Surveillance of Antimalarial Drug Efficacy. Available at: http://apps.who.int/iris/bitstream/10665/ 44048/1/9789241597531_eng.pdf. Accessed March 28, 2017.

14. WHO, 2013. Management of Severe Malaria. Available at: http:// apps.who.int/iris/bitstream/10665/79317/1/9789241548526_eng. pdf?ua=1. Accessed March 28, 2017.

15. Domingo GJ et al., 2013. G6PD testing in support of treatment and elimination of malaria: recommendations for evaluation of G6PD tests. Malar J 12: 391.

16. Singh B, Bobogare A, Cox-Singh J, Snounou G, Abdullah MS, Rahman HA, 1999. A genus- and species-specific nested polymerase chain reaction malaria detection assay for epidemiologic studies. Am J Trop Med Hyg 60: 687-692.

17. Boonma P, Christensen PR, Suwanarusk R, Price RN, Russell B, Lek-Uthai U, 2007. Comparison of three molecular methods for the detection and speciation of Plasmodium vivax and Plasmodium falciparum. Malar J 6: 124.

18. WHO, 2008. Methods and Techniques for Clinical Trials on Antimalarial Drug Efficacy: Genotyping to Identify Parasite Populations. Geneva, Switzerland: World Health Organization, 13-14.

19. Brockman A, Paul RE, Anderson TJ, Hackford I, Phaiphun L, Looareesuwan S, Nosten F, Day KP, 1999. Application of genetic markers to the identification of recrudescent Plasmodium falciparum infections on the northwestern border of Thailand. Am J Trop Med Hyg 60: 14-21.

20. Ariey $F$ et al., 2014. A molecular marker of artemisinin-resistant Plasmodium falciparum malaria. Nature 505: 50-55.

21. Leang R et al., 2015. Evidence of Plasmodium falciparum malaria multidrug resistance to artemisinin and piperaquine in western
Cambodia: dihydroartemisinin-piperaquine open-label multicenter clinical assessment. Antimicrob Agents Chemother 59: 4719-4726.

22. Amato $\mathrm{R}$ et al., 2017. Genetic markers associated with dihydroartemisinin-piperaquine failure in Plasmodium falciparum malaria in Cambodia: a genotype-phenotype association study. Lancet Infect Dis 17: 164-173.

23. Witkowski B et al., 2017. A surrogate marker of piperaquineresistant Plasmodium falciparum malaria: a phenotypegenotype association study. Lancet Infect Dis 17: 174-183.

24. $\mathrm{MOH}$ I, 2013. The National Guideline for Malaria Treatment in Indonesia. Available at: http://p2p.kemkes.go.id/_asset/regulasi/ 65_PMK No. $5 \mathrm{ttg}$ Pedoman Tata Laksana Malaria.pdf. Accessed March 28, 2017.

25. WHO, 2015. Guidelines for the Treatment of Malaria: Third Edition. Available at: http://apps.who.int/iris/bitstream/10665/162441/ 1/9789241549127_eng.pdf?ua=1\&ua=1. Accessed March 28, 2017.

26. Flegg JA, Guerin PJ, White NJ, Stepniewska K, 2011. Standardizing the measurement of parasite clearance in falciparum malaria: the parasite clearance estimator. Malar J 10: 339.

27. Noedl H, Se Y, Schaecher K, Smith BL, Socheat D, Fukuda MM, 2008. Evidence of artemisinin-resistant malaria in western Cambodia. N Engl J Med 359: 2619-2620.

28. Phyo AP et al., 2012. Emergence of artemisinin-resistant malaria on the western border of Thailand: a longitudinal study. Lancet 379: 1960-1966.

29. Tun KM et al., 2015. Spread of artemisinin-resistant Plasmodium falciparum in Myanmar: a cross-sectional survey of the K13 molecular marker. Lancet Infect Dis 15: 415-421.

30. Leang R, Barrette A, Bouth DM, Menard D, Abdur R, Duong S, Ringwald $P, 2013$. Efficacy of dihydroartemisinin-piperaquine for treatment of uncomplicated Plasmodium falciparum and Plasmodium vivax in Cambodia, 2008 to 2010. Antimicrob Agents Chemother 57: 818-826.

31. Davis TM, Hung TY, Sim IK, Karunajeewa HA, llett KF, 2005. Piperaquine: a resurgent antimalarial drug. Drugs 65: 75-87. 